\title{
La Terre, notre bien commun
}

\section{Arnaud Berthoud}

\section{OpenEdition \\ Journals}

\section{Édition électronique}

URL : http://journals.openedition.org/developpementdurable/5693

DOI : 10.4000/developpementdurable.5693

ISSN : 1772-9971

\section{Éditeur}

Association DD\&T

\section{Référence électronique}

Arnaud Berthoud, «La Terre, notre bien commun », Développement durable et territoires [En ligne],

Points de vue (2003-2010), mis en ligne le 07 mars 2008, consulté le 19 avril 2019. URL : http:// journals.openedition.org/developpementdurable/5693; DOI : 10.4000/developpementdurable.5693

Ce document a été généré automatiquement le 19 avril 2019.

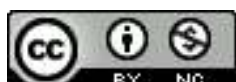

Développement Durable et Territoires est mis à disposition selon les termes de la licence Creative Commons Attribution - Pas d'Utilisation Commerciale 4.0 International. 


\title{
La Terre, notre bien commun
}

\author{
Arnaud Berthoud
}

1 Quelle sorte de chose est la Terre ? Quel sens donnons-nous à ce terme quand nous en usons pour opposer la terre au ciel, pour la faire tourner autour du soleil, pour dire que nous l'avons parcourue et que sa rotondité nous fait revenir sur nos pas, que nous l'habitons dans toute sa surface et toujours en compagnie d'autres êtres vivants, que ses paysages nous enchantent et que sa sauvagerie nous fascine et encore bien d'autres choses auxquelles nous ne pensons pas le plus souvent?

2 Parmi ces choses auxquelles nous ne pensons pas tous les jours quand nous parlons de la Terre se trouvent ces idées qui font néanmoins une partie de notre inquiétude actuelle et que P. Hadot, M. Serres et H. Jonas me semblent avoir mieux exprimées que beaucoup d'autres. Je cite pêle-mêle sans souci de citations précises. La Terre, toute entière découverte, dans tous les sens sillonnée, possédée et maîtrisée à sa surface et dans ses entrailles, cultivée, exploitée, domestiquée et soumise à notre génie prométhéen, ne demande-t-elle pas, dit P. Hadot (2004), que nous soyons à son égard dans une attitude plus orphique, plus amicale et plus contemplative, plus enjouée et plus confiante? Mais pouvons-nous le faire? Nous savons définir la puissance, dit à son tour M. Serres (1990), mais nous ne savons pas penser la fragilité. Or il y aurait urgence à l'apprendre, car la Terre rassemblée et maillée toute entière dans un système global, c'est aussi, comme en tout système unitaire réglé par une loi unique, un système que la destruction peut abattre soudain. Prométhée n'a en effet plus d'espace ou de réserve pour manœuvrer et renouveler ses ruses. La modestie d'Orphée nous est imposée. Nous perdons notre seigneurie. La Terre nous tient et nous oblige, dit enfin H. Jonas (1993). Nous redevenons ses enfants et nous devons en être les gardiens. Au-delà de la responsabilité que la vie nous révèle comme parents ou que la cité nous impose comme citoyen ou homme d'Etat, la Terre nous fait découvrir aujourd'hui une forme de responsabilité à l'égard de l'humanité toute entière. Voilà le fait historique nouveau que l'arrogance de nos techniques, la myopie de nos sciences et la négligence de nos esprits nous feraient volontiers oublier. 
Quelle sorte de chose est donc la Terre lorsque nous usons de ce terme sous ces formes variées et que nous tentons de nous hisser au niveau de ce fait historique nouveau et de notre inquiétude à son sujet ? Je propose cette réponse générale : la Terre nous apparait comme ce qui a été, est et sera toujours la chose la plus vaste à laquelle s'attache notre bien commun, parce qu'elle est le support, le fondement ou la base visible d'une vie que chacun éprouve intérieurement, selon un lien immédiat avec quelques semblables et comme le bien dont nous savons, comme le disait Maître Eckhart (1948, p.148), que « de tous les biens aucun ne nous est plus cher et plus désirable ». C'est cette réponse que je tente de développer en précisant successivement sous l'inspiration d'Aristote quelques traits relatifs au bien commun dont la Terre serait le support le plus vaste et sous l'inspiration biblique quelques remarques relatives au lien vivant qui nous attache à nos semblables et dont la Terre serait la base visible la plus claire. Je dirais alors que dans le premier cas la Terre est saisie comme un objet économique et que dans le second cas la Terre est saisie comme un objet religieux. J'essayerai dans la conclusion de rapporter ces deux types de propos l'un à l'autre.

Que dire d'un bien commun lorsqu'il s'attribue comme prédicat à une chose et qu'il prend ainsi la forme d'un bien extérieur ou d'une richesse? On peut distinguer quatre caractères. Le bien commun ou la richesse commune relève d'abord d'un donné avant toute prise et toute production. Le bien commun ou la richesse commune est ensuite reçu, accueilli et mesuré par un acte collectif dont le nom est le travail. Le bien commun ou la richesse commune donne lieu à propriété sous l'exigence de justice. Enfin le bien commun ou la richesse commune est consommé selon une jouissance singulière propre à chaque individu. Ces quatre caractères économiques constituent autant de parties de ce qu'on peut appeler une philosophie économique de la richesse commune. C'est ainsi que la Terre comme la chose la plus vaste à laquelle s'attache notre bien commun est un objet économique et relève d'abord d'une philosophie économique.

Nous ne pourrions pas tenter de vivre bien, comme nous cherchons manifestement à le faire, en dépit de nos échecs répétés, si le bien ne nous était pas d'emblée donné comme une fin orientant nos vies et s'imposant à nos actions. De la même manière, nous ne pourrions pas tenter de vivre ensemble le mieux possible si le bien qui oriente nos vies et qui s'impose à nos actions n'avait pas d'emblée le caractère d'un bien commun. Ce n'est pas de notre individualité que vient l'idée qu'il nous convient à chacun de vivre ensemble et que la vie commune est orientée elle-même par un bien.

Or nos actions communes se distinguent d'abord selon les deux grandes dimensions de l'économie et de la politique qui donnent deux formes extérieures au bien défini par l'éthique. Il faut donc concevoir que le bien commun extérieur qui s'impose à l'existence de la communauté de vie dans les actes de consommation, de production et de distribution est autre que le bien commun qui s'impose à la communauté politique. Pour la politique, le bien commun extérieur qui nous est d'emblée donné est le Droit, la justice, la paix et le bonheur de parler ensemble et de se comprendre selon la diversité des personnes. Pour l'économie, le bien commun extérieur n'est rien d'autre que le bien-vivre ensemble ou la richesse commune dont la mesure propre est l'économie elle-même. Les richesses sont les objets extérieurs du besoin sur lesquels une communauté exerce la mesure propre de son bien-vivre ou de son économie.

7 La mesure propre du bien-vivre ou l'économie d'une communauté lui est ainsi donnée comme lui sont donnés les premiers objets du besoin. Il n'y a jamais un état zéro de vide où rien ne serait donné au point de départ d'une communauté d'êtres vivants, comme si 
tout devait être produit sur une table rase et comme s'il fallait inventer une définition du bien-vivre ou comme s'il fallait construire dans l'ordre politique la diversité des personnes, la paix, le Droit et le bonheur de se comprendre. De même qu'il y a toujours déjà un bien commun à la base de l'existence politique et que faute de le poser comme donné au départ on ne pourrait plus jamais le trouver par la suite, de même il y a toujours déjà des richesses ou des biens - qui sont donnés comme objets selon l'idée d'une mesure du bien-vivre ou l'idée d'une économie. La Terre est notre bien commun en ce sens. Nous n'avons pas produit la Terre et elle n'est pas à proprement parler un produit que nous pourrions transformer, refaire ou même déconstruire. Elle est d'emblée notre lieu de vie où se déploient les premiers services de nos semblables et où s'offrent quelques biens matériels, qui forment tous ensemble nos premières richesses. La Terre est le premier objet de toute économie.

8 Si la Terre nous est ainsi donnée à titre de premières richesses et de première économie, il s'agit maintenant de la recevoir. A quelle activité et à quelle forme d'expérience rapporter cet accueil ou cette réception? Répondre à cette question, c'est dire aussi comment la richesse primitivement donnée est reconnue comme le bienfait ou le bénéfice d'un moment du temps contre le temps qui l'use et l'entraîne vers sa disparition. Le travail est cette activité et cette expérience. Dominée par la valorisation sans limite de la production et par l'ivresse des inventions mécaniques, l'époque moderne oublie souvent que le travail n'est pas seulement un acte productif et ne désigne pas seulement une activité de transformation d'une matière donnée dont le résultat objectif est un produit. Dans la plupart des sociétés, aux termes correspondant à ce que nous appelons aujourd'hui «travail en général » est associée une expérience commune spécifique de la mesure du temps qui passe. Travailler, en ce sens, c'est lier le temps de l'objet qui change et qui s'use au temps du corps qui souffre ou c'est compter les heures anonymes du temps astronomique de la transformation de l'objet en heures de peine vécues ensemble. L'usure des choses et des services est mesurée par l'usure des corps. Cela vaut pour tous les objets dont nous usons et que nous consommons. Le travail n'est pas de l'autre côté de la consommation, comme le rappelle $\mathrm{H}$. Arendt contre la pensée courante (1961).

9 Nos machines ou les outils de notre travail productif fonctionnent dans le temps, mais n'éprouvent pas le temps qui passe comme douleur. Leur exercice est sans mesure interne. Seul le travail proprement humain saisi sous son aspect subjectif enveloppe une mesure interne du temps et seul, en conséquence, le travailleur collectif recueille le sens de l'économie. Inversement, une société qui n'a pour valeur que la production et le travail sous son aspect exclusivement productif ne connaît plus le sens de l'économie véritable. Accueillir ce qui est donné comme richesse par son travail, ce n'est pas d'abord s'en emparer pour en modifier précipitamment la forme - attitude productiviste ou prométhéenne. C'est plutôt, comme le langage le dit avec profondeur, en prendre toute la mesure et découvrir la valeur et la fragilité de son bienfait - attitude plus orphique. Accueillir la Terre qui nous est donnée comme bien commun et première économie par notre travail collectif, c'est en devenir les gardiens vigilants et économes qui éprouvent les rythmes de vie et les temps d'usure de chaque bien.

La Terre nous est donnée en commun comme une richesse première que nous accueillons ensemble par notre travail comme un bienfait dans le temps qui passe. Quel sens donner à cette "communauté » constituée par le don et son accueil ? La terre est à tous les hommes et pour tous les hommes : cela veut-il dire que chaque homme pourrait tour à tour affirmer de la Terre qu'elle lui appartient en propre et qu'il peut en devenir 
individuellement propriétaire - forme distributive de la propriété commune - ou cela veut-il dire que la Terre appartient aux hommes pris tous ensemble collectivement forme indivisible de la propriété commune? La question de l'appartenance ou de la propriété n'a pas cessée d'être débattue depuis la discussion qui oppose Aristote à Platon sur le sens du communisme ou de la communauté des biens et des services. Je me range du côté des arguments présentés par Aristote dans le livre II de «La Politique » en les adaptant au thème de la Terre. "Commun», selon Aristote, ne se dit ni exclusivement d'une propriété attribuée à chacun de manière distributive, quelle que soit la nature du bien, faisant par exemple d'un champ, d'un vêtement ou du service d'une femme la propriété de tous les hommes pris un à un, ni exclusivement d'une propriété attribuée à tous selon une unité indivisible, quels que soit les différents moments de la propriété. Il faut au contraire tenir compte de la nature des biens et des services - ce qui semble évident - et il faut plus encore tenir compte des moments de la propriété - ce qui est plus subtil. Or il y a deux moments essentiels dans la propriété - la possession et l'usage - à situer au sein d'une séquence faite elle-même de quatre notions distinctes par lesquelles le bien commun ou la richesse commune se décompose : le donné, la réception du donné, la propriété - prise de possession et usage - et la jouissance.

11 Revenons sur les deux premières notions. Le donné et la réception du donné sont communs au sens d'une unité indivisible : la Terre est la richesse première de l'humanité ; personne ne peut en être exclu et nul ne peut prétendre en être le gardien ou l'économe exclusif par son travail ; l'exercice du travail nous attache les uns aux autres autant sous sa forme objective que sous sa forme subjective - tradition aristotélicienne reprise sur ce point par Marx, contre les prétentions individualistes issues de la doctrine du travail chez Locke. Forme objective du travail : forme toujours collective, comme en témoigne l'outil inséparable du travail et dont la première apparition remonte à la main d'autrui. Forme subjective du travail : expression de notre commune humanité, comme l'indique à sa manière la mesure en temps de peine dont chacun partage avec tous le même sens.

12 Les deux moments suivants de la possession et de l'usage composent à proprement parler la notion de propriété. La propriété est le nom d'une relation qui rapporte un ensemble de richesses à un être de besoin pourvu d'une intelligence ou d'une raison. C'est l'exercice de ce pouvoir de la raison sur les biens que le Droit protège par «le droit de propriété ». Le droit de propriété s'exerce, d'une part, comme possession mettant le bien à l'abri de l'instabilité au long de son temps d'usure et, d'autre part, comme usage faisant de la richesse un moyen légitime en vue d'une fin - fin qui est la jouissance proprement dite et l'élément dernier du bien commun ou de la richesse commune sur laquelle on reviendra dans un instant. C'est sur les deux moments de la possession et de l'usage que porte le cœur de la difficulté opposant Aristote à Platon.

13 La possession dans le temps et la mise en relation du moyen à la fin par l'usage doivent-ils être l'un et l'autre communs et que veut dire en chaque cas " commun »? Comment ces deux actes se conforment-ils chacun au mieux au fait de la richesse commune donnée et accueillie antérieurement par l'unité indivisible de l'humanité au travail ? La conformité au bien définit ici la justice. Le bien d'abord, la justice ensuite. Aujourd'hui, on dit volontiers que cet ordre de préséance définit la forme téléologique de la morale. Aristote considère que la justice n'est pas nécessairement respectée et le bien commun honoré, comme le croit Platon, en faisant de la possession et de l'usage des actes communs exercés par chacun tour à tour de manière distributive. 
Il faut commencer par l'usage. Pour la tradition aristotélicienne, l'usage doit rester commun de manière distributive, parce que la Terre est donnée et reçue pour que tous en vivent chacun à sa manière et que personne ne soit exclu de l'humanité dont la Terre est la demeure. L'accaparement privé qui laisserait des hommes dans la pauvreté et hors de la communauté humaine est en cela injuste. Chacun a donc un droit d'usage par l'intermédiaire de l'échange, de la répartition, du prêt ou du don, qui sont les quatre formes de la distribution. La prodigalité ou la parcimonie dans l'une ou l'autre de ces quatre formes de distribution sont des excès dont le juste milieu définit la juste mesure de l'usage économe du bien. On parlera alors d'une doctrine du juste prix dans l'échange, du mérite justement reconnu dans le partage, du taux d'intérêt refusé ou limité dans le prêt et de l'assistance des plus démunis par le don. Ici, la pensée aristotélicienne et son héritage thomiste sont plus précis que la pensée de Platon, mais ils n'en différent pas profondément.

Ensuite, la possession. C'est ici qu'Aristote et Platon divergent le plus. Aristote considère que l'attachement, le soin, la sollicitude et l'amour sont plus profonds quand ils s'exercent de manière exclusive. Un partage du bien commun en possessions privées et séparées - champs, forêts, mines, lieux de vie, services mutuels etc. - peut ainsi assurer une stabilité plus grande et satisfaire ce qu'il appelle le sentiment ordinaire de propriété, «car il y a dans l'homme deux mobiles prédominants de sollicitude et d'amour, le sentiment de la propriété et l'affection exclusive » (traduction Tricot, 1262b 22). La Terre commune n'implique donc pas nécessairement la possession commune. En définitive, la justice qui honore le bien donné sous forme de Terre commune se scande de la manière suivante : donné commun et réception commune au sens d'une unité indivisible, usage commun au sens d'une unité distributive et possession privée ou commune selon la nature des biens.

Tout bien a pour fin ultime la jouissance - le « frui ». La jouissance du bien est le moment où le bien prend la forme sensible du bon, du plaisir et du bonheur. Un bien qui ne serait pas connu sous cette forme éminemment subjective de la jouissance, du plaisir et du bonheur perdrait son sens. S'il est vrai que le bien s'attache à des objets comptés comme richesses et sous la juste mesure du bien vivre ou de l'économie, alors il faut qu'il y ait une forme spécifique du bonheur dans le don, la réception, la possession et l'usage, distincte de la forme du bonheur dans l'existence politique. Ou encore, il faut qu'il y ait une jouissance dans la consommation de la Terre qui ne doit rien à ce qui constitue la paix, la justice ou le bonheur de la conversation avec les autres. On peut appeler amour de soi-même cette jouissance. C'est le moment le plus augustinien d'une philosophie de la Terre.

La jouissance se caractérise par quatre traits. D'abord, «frui » se distingue de «l'usus ». User, c'est amener l'objet jusqu'aux portes de la subjectivité et de la vie, alors que jouir ou se réjouir, c'est plutôt lier l'objet à l'amour de soi-même. La richesse, voilà ce dont on fait usage comme moyen ; la vie en soi-même ou soi-même comme être vivant, voilà ce dont on se réjouit et qu'on aime en s'aimant soi-même. En second lieu, la vie dont on se réjouit et dont on s'aime est la vie dans son caractère extérieur et terrestre, la vie qu'on aime vivre comme vivant en un lieu et en un temps, parmi ses semblables à qui l'on montre d'ailleurs volontiers son contentement et sa joie - comme le rappelle si volontiers A. Smith. En troisième lieu, cet amour de soi se définit par opposition à deux formes contraires: l'ascèse selon laquelle la richesse n'est plus un moyen de se réjouir aujourd'hui, mais seulement un moyen d'accumuler en remettant sans cesse à demain le 
moment de la jouissance ; l'amour-propre selon lequel chacun ne connaît de soi-même et n'aime en soi-même que ce qui le distingue de l'autre. De fait, et c'est le dernier trait, il n'y a pas d'autre que soi dans le moment de la jouissance de sa propre vie ou, pour le dire autrement, il n'y a pas d'amour d'autrui dans la phase finale de la consommation. La Terre est commune dans le don et l'accueil selon l'unité indivisible de l'humanité. Elle est commune dans l'usage selon une notion distributive de la communauté. Elle peut être privée dans sa possession. Mais elle est personnelle et singulière dans le moment de la jouissance, qui est aussi le moment où chacun se réjouit d'être le seul à vivre sa vie. C'est ainsi que la Terre tour à tour commune, privée et singulière selon les actes économiques qui en font un objet est proprement un objet économique.

Est-ce que la question posée de savoir quelle sorte de chose est la Terre a reçu maintenant une réponse suffisante? La Terre serait la première richesse donnée aux êtres humains et reçue à travers leur travail, une propriété commune partagée selon des règles de justice précisant ce qu'il en est de la possession ou de l'usage et la source dont se tire pour chacun la jouissance et l'amour de sa vie. Mais est-ce assez de désigner la Terre de cette façon - comme objet de consommation et premier objet de toute économie ? La Terre n'appartient pas directement au domaine des objets politiques ou esthétiques: ni cité régie par un Droit, même si le Droit peut en faire son horizon, ni chose de la nature ou œuvre d'art proposé à notre sentiment du beau, même si son spectacle devient aujourd'hui possible. Mais n'y a-t-il rien d'autre ? N'y a-t-il pas un discours poétique, mythologique et théologique sur la Terre qui en fait un bien commun et un bien commun extérieur défini tout autrement que par les catégories de l'éthique, de l'économie, de la politique et de l'esthétique ? De fait, c'est par les hymnes et les prières, les cosmogonies ou les récits de la création que la Terre entre d'abord dans nos savoirs. Avant d'être saisie comme première richesse, la Terre a été et reste pour nous la première chose dont la stabilité nous étonne, le milieu primitif de la vie dont l'énigme confond nos esprits et la matière d'un temps qui soulève mystérieusement notre joie et notre attente? N'est-ce pas cela que tentent de dire les poésies, les mythologies ou les théologies sur la Terre? Elle serait tout à la fois l'exemple même de l'ordre ou des lois nous enseignant la mesure et la raison, la figure de notre ignorance profonde sur la vie d'où nous tirons notre humilité ou notre compassion pour tous les vivants et une sorte d'invitation joyeuse à nous lier toujours davantage les uns aux autres dans l'attente d'un dernier jour. M. Serres propose cette définition minimum de la religion comme lien et pacte de tous les hommes avec la Terre et dont le contraire serait "la négligence». "Par les contrats exclusivement sociaux, dit-il, nous avons laissé le lien qui nous rattache à la Terre...Qui n'a point de religion ne doit pas se dire athée ou mécréant, mais négligent » (1990p.81). En reprenant sous cette forme modeste la notion de religion, on dira que la Terre n'est pas seulement un objet économique, mais qu'elle est aussi et d'abord un objet religieux et, comme tel, une fois encore notre bien commun. Notre bien commun ne serait donc pas uniquement le bien-vivre et la richesse première dont s'occupe une philosophie économique de la Terre. Il serait fait aussi de la raison et de la mesure, de l'énigme de la vie et de l'attente d'une joie collective à quoi la Terre nous lie par un lien sans doute plus fort ou plus primitif que tous les liens de l'économie. C'est en cela aussi que nous sommes des enfants de la Terre. Je propose maintenant quelques remarques en situant la Terre sous trois traditions religieuses différentes en terminant par la tradition juive et biblique qui inspire cette seconde partie. 
19 1. A quoi nous lie la Terre et de quelle manière fait-elle de nous ses enfants? Il faut sans doute dire - première tradition - que la Terre nous attache à l'ordre et aux lois qui la constituent et qu'elle fait de nous à ce titre des êtres dont la conscience la plus intime est d'être à soi-même comme le miroir d'un ordre et le produit d'une nature. Nous sommes des êtres naturels. La nature agit comme un sage artisan ou comme un artiste sachant éviter le trop et le trop peu et donnant sa mesure aux choses qu'elle fait apparaître. La Terre est notre demeure en étant d'abord un abri ou une protection défini et proportionné à notre nature. Nous sommes sur la Terre et sous le ciel, protégés du chaos ou du désordre total. Le temps a son rythme régulier sur lequel nous pouvons compter; chaque nuit a son terme et laisse place au jour qui nous rassure ; les saisons se succèdent et nous apprennent la variété de nos humeurs ; l'eau, la terre, le feu et l'air donnent leur mouvement propre à nos amours et à nos haines; l'espace a ses limites définies qui nous gardent des confusions du haut et du bas, du proche et du lointain ; il y a les couleurs, il y a les qualités et il y a les nombres; chaque chose a sa place, son rang et son temps. Toute la Terre, faite ainsi selon l'art divin de la nature en parties ou en éléments séparés, se propose à notre imitation. Elle nous enseigne l'art et les techniques pour nous puissions saisir à notre tour les choses et les mesurer aux besoins de notre corps. Notre corps nous apparait lui-même comme une terre. Si celle-ci est un ordre, une organisation ou un organisme, une sorte de cosmos qui réfléchit un cosmos plus large, alors notre corps est lui aussi un cosmos ou un microcosme. La Terre et notre corps se mesurent l'un l'autre. Que serions nous ensemble et comment pourrions nous chacun se reconnaitre si nous n'avions pas pour abri cette Terre qui nous enveloppe et nous protège de ce que les Grecs appellent "l'apeiron", l'illimité ou la démesure ? Quelle humanité serions nous si la Terre était sans loi, sans nature ou sans mesure ? La pensée grecque achève son parcours religieux dans la célébration stoïcienne du Logos. Le Logos ou la Raison assiste la Terre et guide les hommes. Etre homme, c'est avoir sous la forme de la Terre la mesure ou la raison pour bien commun.

2. La Terre nous lie encore d'une autre manière. Elle n'est pas seulement un cosmos et une figure de la Raison. Elle est aussi une Terre habitée et la demeure du vivant. Or de tous les êtres vivants qui peuplent la Terre, les hommes sont sans doute les seuls à se connaître comme les miroirs de l'ordre et de la mesure terrestre, mais ils ne sont pas les seuls à avoir de leur vie un sentiment ou une appréhension. Mais comment les autres êtres vivants qui côtoient nos temps et nos espaces ressentent-ils le fait de vivre? Cela, nous l'ignorons. Nous sommes ainsi environnés et accompagnés tout au long de notre parcours sur la Terre d'une multitude d'êtres qui nous sont à la fois contemporains et situés de l'autre côté d'une même barrière invisible. De ce côté, il y a pour chaque homme un ou plusieurs semblables avec qui partager la même connaissance et la même énigme d'être un vivant. De l'autre côté, il y a les oiseaux, les poissons et toutes les bêtes des forêts, des montagnes et des plaines, de taille, de forme et de mœurs différentes, plus ou moins fragiles ou féroces, plus ou moins gracieux ou hideux, tous enfermés dans la même réserve ou dans le même silence sur la vie qui est la leur. Les animaux se taisent et ne nous disent pas plus qui ils sont que ne font les plantes ou les fleurs. "La rose est sans pourquoi, fleurit parce qu'elle fleurit, sans souci d'elle-même, ni désir d'être vue » dit le poète. Sans désir? Mais qu'en savons-nous? Le silence des animaux sur leur vie nous renvoie à notre propre ignorance sur la nôtre. L'énigme de notre vie s'approfondit en énigme générale sur le vivant. On pourrait dire que le cosmos ne se dévoile pas dans le logos sans se voiler plus encore dans la vie ou que la Raison qui nous est donnée comme 
une lumière pour réfléchir l'ordre ou les lois n'éclaire qu'une partie fort réduite d'une obscurité beaucoup plus profonde. Nous savons beaucoup de choses et en même temps nous ne savons presque rien. Nous nous faisons les maîtres des animaux parce que nous connaissons les lois de notre demeure commune. Mais nous ne savons pas répondre ni pour nous ni pour eux au pourquoi de la vie que nous vivons pourtant chacun ensemble. La maitrise de notre raison fait notre grandeur. Notre ignorance sur le sens de la vie fait notre faiblesse et nous met en quelque sorte et si nous le voulons bien, à l'école des autres vivants, de leur mutisme et de ce qui nous semble être le mystère de leur paix et de leur humilité. Par eux, nous apprenons une autre forme de limite. La Terre n'est pas seulement de l'autre côté du désordre ou du chaos et donnée comme cosmos à notre connaissance, comme la religion grecque nous l'a si bien apprise. La Terre nous est aussi donnée, à nous et à tous les vivants comme un lieu mystérieux au-delà de la limite de notre raison. Voilà sans doute ce que disent les plus anciennes religions. Notre bien commun dont la Terre est la figure n'est pas seulement la raison. Il est aussi et paradoxalement l'ignorance. Sous l'effet du témoignage silencieux de ceux qui vivent avec elle et comme autre qu'elle, l'humanité est aussi constituée en-deçà de la raison par l'ignorance profonde de ce qu'elle est. C'est à cette source que s'abreuvent les formes sacrées de la compassion et du respect de la vie dans le bouddhisme.

21 3. La Terre nous lie et nous constitue encore sous une troisième forme. Elle ne nous réunit pas seulement dans la même demeure par la Raison et par l'ignorance ou par la connaissance de la mesure et par l'énigme de la vie. Elle nous attache à la même joie, la même jubilation ou le même bonheur collectif. L'idée d'une Terre heureuse est sans doute un trait particulier de la tradition juive reprise dans la spiritualité chrétienne. "Que les cieux se réjouissent, dit le psalmiste, et que la terre soit dans l'allégresse, que la mer retentisse avec tout ce qu'elle contient, que la campagne s'égaie avec tout ce qu'elle renferme, que tous les arbres des forêts poussent des cris de joie, devant Yahvé » (Ps. 96/11). Ce qui rattache la Terre à la joie ou ce qui fait de notre demeure commune un lieu de bonheur, c'est le fait que la Terre ait été créée et que le résultat de cet acte ait été qualifié de "bon et de très bon " par la joie de son auteur (Genèse $1 / 31$ ). Cette parole de jubilation qui s'inscrit au cœur de la Terre ne se retrouve ni dans la raison immanente à la Terre de la religion grecque, ni dans l'énigme de la vie terrestre des religions anciennes et du bouddhisme. Or cette joie essentielle ou constitutive de toute l'étoffe de la Terre, c'est aussi ce qui fait apparaitre tout mal ou tout malheur qui la frappe comme un accident ou comme une sorte d'irréalité impensable. La Terre peut se trouver profondément blessée, l'humanité peut voisiner de manière infiniment troublante avec le chaos ou le néant, la vie peut s'éprouver comme douleur et son énigme redoubler sous toutes les formes de souffrance, la mort peut sembler avoir le dernier mot : le fait de la création bonne et très bonne par un Dieu qui ne renie pas son œuvre est au principe de la foi juive et chrétienne. La nature sous la clarté des lois et des mesures ou la vie qui se retient dans l'obscurité de son énigme sont des états. La création n'est pas un état ; elle est un événement. La Terre heureuse est un événement de l'histoire du Dieu Yahvé avec les hommes. Il y a ici une troisième forme de limite : non plus entre la mesure et le chaos, ni entre la raison et l'ignorance de la vie, mais entre le plan divin et l'histoire terrestre ou profane des hommes. Comme telle, l'histoire profane n'est pas une création continuée, mais se déroule selon son propre temps. Il y a sans doute un passé antérieur à la Terre et à l'humanité qui en constitue une sorte de mémoire et il y a surtout un futur dont la présence constitue en chaque homme et en tout vivant son soupir ou son attente. La Terre est une demeure d'attente parce qu'elle est un événement et que tout événement 
enveloppe avec sa nouveauté l'attente d'un événement ultérieur. L'événement ultérieur attendu par toutes les créatures est le jour nouveau et le sabbat définitif où la joie effacera toutes les blessures et toutes les larmes versées à la surface de son histoire.

1- Quelle sorte de chose est la Terre ? La question n'a sa portée qu'en dépassant le plan de la science. L'astronomie ou les sciences de la Terre nous apprennent des faits et nous rendent attentifs à des régularités, des causes ou des lois. Mais la Terre elle-même n'est pas une chose dont une science pourrait donner le sens. Le sens relève d'une autre sorte d'interrogation, qu'on peut dire philosophique ou religieuse selon les cas. Affirmer de la Terre qu'elle est la chose la plus vaste à laquelle s'attache notre bien commun ou la demeure de notre vie vécue chacun avec quelques-uns de nos semblables répond à une question sur son sens. Ce n'est pas une proposition scientifique.

2- Comme bien commun et demeure d'une vie vécue chacun avec quelques semblables, la Terre semble d'abord un objet économique. Elle est notre richesse première, donnée à tous, reçue de tous dans le travail, possédée et mise en usage par tous et consommée à même la jouissance par chacun de sa vie singulière. Mais elle est encore autre chose qui dépasse l'économie et qui ne relève ni de la politique ni de l'esthétique. Elle est un objet religieux dont les différents caractères sont autant de traits qui nous lient étroitement les uns aux autres dans une notion élargie d'humanité. L'humanité des enfants de la Terre n'est pas faite seulement de gardiens économes; elle est faite aussi de poètes ou d'être religieux dont le bien commun est tout à la fois la raison, l'ignorance et la joie. Ce sont tous ces traits qu'il nous faut mobiliser pour tenter de nous hisser au niveau du fait historique nouveau et répondre à notre inquiétude. Attitude orphique - P. Hadot; pacte sacré avec la Terre - M. Serres; Morale nouvelle de la responsabilité - H. Jonas. Il nous faut devenir en même temps économe, sujet moral et poète de la Terre.

3- La notion même de richesse première à son tour n'est pas une notion que la science économique des temps modernes peut développer. S'il est juste, en effet, de définir notre science économique à partir de la science physiocratique et classique comme une science à la recherche des conditions de reproduction de la richesse globale d'une société donnée, dont la théorie néo-classique n'est qu'une partie inscrite sous le modèle de l'individualisme marchand, la notion d'une richesse première donnée sous forme d'un bien commun et offerte à la jouissance de chacun tombe naturellement en dehors de son champ. Une science de la reproduction fait nécessairement de la consommation ou de la jouissance dans la possession et l'usage de la richesse un simple moment d'une production qui constitue pour elle l'acte fondamental et premier de toute l'économie (A. Berthoud, 2005). Sous cette perspective, le don ou le donné ne peut venir qu'après et au même rang que les actes de distribution. Or sans le don et le donné, la Terre n'est plus saisie comme un objet singulier. Elle n'est qu'un ensemble indéterminé de facteurs de production exploitables. Pour traiter de la Terre comme la chose la plus vaste à laquelle s'attache notre bien commun, il faut donc sortir de la science économique des temps modernes.

4- S'il est juste de considérer que les hommes ont très probablement tiré de la Terre leur premier moyen de vivre avant d'accéder au bien commun de la raison, de l'énigme et de la joie, on doit dire en un autre sens que la Terre dans la pensée des hommes a d'abord été un objet religieux avant d'être traité comme un objet économique ou que les religions de la Terre viennent avant la philosophie économique de la Terre. Cela suggère plusieurs choses. C'est la Terre économe et ses mesures - tradition grecque - qui inspireraient nos notions d'économie et de travail. C'est la Terre vivante habitée d'énigme et de compassion 
qui servirait de fond à l'utilitarisme et la justice sociale. Et c'est la Terre remplie de joie et dans l'attente d'une joie plus grande encore qui légitimerait notre volonté d'agir ensemble sous le don d'une richesse première pour que chacun puisse à la fin s'aimer soimême comme vivant. Nous serions poètes avant d'être économes et nous ne serions pas économes sans être aussi poètes. L'agent économique véritable, oublié par la science économique moderne, ne serait pas seulement un sujet moral. Il serait aussi à sa façon un être religieux. Je rejoins ici à ma manière le fond durkheimien et maussien des amis d'A. Caillé.

5- La Terre est la chose la plus vaste à laquelle s'attache notre bien commun. Nous, c'està-dire tous les hommes, l'humanité, gardiens économes ensemble de la même demeure et ensemble enfants ou poètes des mêmes religions de la Terre. Faut-il dire aussi citoyens ensemble de la même unité politique ? Voilà sans doute la question la plus difficile. Le fait historique nouveau semble enlever à la politique la prééminence qu'on lui accorde le plus souvent depuis Hegel jusqu'à Léo Strauss, E.Weil ou H. Arendt et du côté des sociologues P. Bourdieu ou M. Gauchet. Il semble qu'il faille dire maintenant : économie et religion d'abord, politique ensuite et de manière problématique. Dans la perspective de la Terre et sous le point de vue de l'humanité dont elle est la demeure et le bien commun le plus vaste, ce n'est pas la cité, l'Etat et ses dispositifs juridiques contraignants qui semblent se trouver au cœur de l'histoire des hommes. Au contraire, la politique semble trahir l'idée plus profonde d'une Terre comme demeure, maison ou foyer dont tous les habitants sont des enfants et des frères. L'humanité n'est pas un concept familier de la politique. M. Serres disait qu'il nous fallait laisser la puissance et apprendre à penser la fragilité. Il faut sans doute dire aussi qu'il nous faut apprendre à penser la politique autrement et en dehors des seules notions de maîtrise et de liberté léguée par la pensée grecque et Aristote en particulier. Il nous faudrait peut-être inventer, pour donner sens à la Terre, une notion nouvelle de politique dont la base serait la faiblesse, le service et la fraternité. Mais qui faut-il alors invoquer ici pour nous guider? Je ne sais et termine ces lignes sur cet aveu d'ignorance.

\section{BIBLIOGRAPHIE}

Arendt H. (1961) Condition de l'homme moderne, Calmann-Lévy

Berthoud A. (2005) Philosophie de la consommation, Presses Universitaires du Septentrion

Maître Eckhart (1948) Traité et Sermons, Aubier

Hadot P. (2004) Le voile d'Isis, Gallimard

Jonas H. (1993) Le Principe de Responsabilité, Edition du Cerf

Serres M. (1990) Le Contrat Naturel, Fr. Bourin 


\section{RÉSUMÉS}

La Terre nous fait découvrir aujourd'hui une forme de responsabilité à l'égard de l'humanité toute entière. Quelle sorte de chose est donc la Terre lorsque nous usons de ce terme sous ses formes variées et que nous tentons de nous hisser au niveau de ce fait historique nouveau et de notre inquiétude à son sujet? Je propose cette réponse générale : la Terre nous apparaît comme ce qui a été, est et sera toujours la chose la plus vaste à laquelle s'attache notre bien commun, parce qu'elle est le support, le fondement ou la base visible d'une vie que chacun éprouve intérieurement, selon un lien immédiat avec quelques semblables et comme le bien dont nous savons, comme le disait Maître Eckhart (1948, p.148), que « de tous les biens aucun ne nous est plus cher et plus désirable ». C'est cette réponse que je tente de développer en précisant successivement sous l'inspiration d'Aristote quelques traits relatifs au bien commun dont la Terre serait le support le plus vaste et sous l'inspiration biblique quelques remarques relatives au lien vivant qui nous attache à nos semblables et dont la Terre serait la base visible la plus claire. Je dirais alors que dans le premier cas la Terre est saisie comme un objet économique et que dans le second cas la Terre est saisie comme un objet religieux. J'essayerai dans la conclusion de rapporter ces deux types de propos l'un à l'autre.

\section{INDEX}

Mots-clés : bien commun, terre

Keywords : Common good, earth

\section{AUTEUR}

\section{ARNAUD BERTHOUD}

Arnaud Berthoud est économiste et philosophe, professeur émérite à la Faculté des sciences économiques et sociales, Université Lille1. Ses recherches s'inscrivent dans le domaine de la philosophie économique, en particulier la philosophie de la consommation, sujet sur lequel il a publié un ouvrage en 2005 (Philosophie de la consommation, Presses Universitaires du Septentrion). 